\title{
Study on Comprehensive Evaluation Index System for Education E-government Performance of College and University
}

\author{
Lijuan Ye, Fang Yu and Jiaming Zhong \\ Xiangnan University, 423000 Chenzhou Hunan, China
}

\begin{abstract}
First, it sets forth the connotation of education E-government performance evaluation in college and university ,Then it illustrates its design principles as directive, complete, scientific, feasible, and development. It also puts forward the construction process, including dispersion, convergence, test, and verification. Meanwhile, it determines the framework for this index system, including four primary indexes, which are information infrastructure, application system, income and benefit, insurance system, as well as eighteen secondary indexes, namely possession quality of computer products per capita, construction of office and business application system, hardware, software, personnel training, and views and talent environment, etc. By making use of analytic hierarchy process, it determines the weight of primary index and the secondary index, thus constructing a relatively complete comprehensive evaluation index system for education E-government performance of college and university.
\end{abstract}

\section{Introduction}

The construction of education E-government is an important part of education information and the national E-government affairs, and it is an important approach of advancing the educational modernization. To transform the working style and method, as well as to further improve the work quality and efficiency, current universities and colleges are advancing the e-government construction now, and with some results. But in all, it still has some problems, such as large input while small output, high investment while low efficiency. Therefore, e-government construction in colleges and universities is far from adapting to the requirements of educational reform and development in China. International practices and studies have shown that the e-government project is highly risky and extremely possible to fail, so many research institutions, consulting companies, and scholars in western countries have studied on the e-government performance evaluation. The study on e-government performance evaluation can be divided into five international models, namely: performance focusing on government websites, such as American Brown University, Accenture Consulting Company; technical index of infrastructures, such as IBM Company; comprehensive index system of software and hardware, such as the United Nations and American Administrative Society; network performance in the whole society, such as International Development Center for Harvard University; basic evaluation standards, such as OECD (Organization for Economic Cooperation and Development) ${ }^{[1-4]}$.

Some domestic research institutions have evaluated the portal websites of central government and local governments, and many experts and scholars have conducted a lot of researches on the e-government evaluation index system and methods in line with the characteristics of domestic e-government construction. Zhang Chengfu, Tang Jun (2005) put forward that the e-government performance can be divided into three levels of output, result, and influence ${ }^{[5]}$. Yang Yunfei, Bai Qing (2004) established an e-government evaluation index system including five items, which are "electronic concentration, electronic safety, electronic 
management, electronic service, and electronic decision" ${ }^{6]}$. Peng Xizheng (2005) maintained that the main factors of e-government performance evaluation are: evaluation index system, index evaluation standard, index weight, comprehensive scoring methods, and data collection method ${ }^{[7]}$. Ma Lianjie, Hu Xinli, Zhang Xiaolian et. al. (2005) designed a set of e-government project evaluation index from the perspective of cost benefit ${ }^{[8]}$. Liu Teng, Liu Ronghui, and Zhao Peng (2004) established internal and external index systems, as well as six subsystems ${ }^{[9]}$. Wang Xiezhou, Chen Yanhong (2004) established the evaluation index system for e-government performance from the quantity, quality, utilization, and development potential of e-government information resource construction ${ }^{[10]}$.

In all, the e-government performance evaluation in China is still at the initial stage, and the construction of scientific e-government performance evaluation system is at the exploratory period.

\section{Concept of Education E-government Performance Evaluation}

\subsection{Performance}

Performance is the work expression and business result of the organization or its subsystems (department, procedure, work teams and employees), which reflects the following meanings: first, it is the expression of subsystem during the work process; second, it is the business result of subsystems.

\subsection{Education E-government Performance in College and University}

According to the meaning of "performance" discussed in the previous part, the education e-government performance in college and university can be summarized in two aspects: process and result, namely the e-government operation process and result for school education. The operation process is to improve the school targets through effectively allocating information resources; the operation result is the contribution degree of realizing enterprise targets by making use of education e-government results.

Therefore, education e-government performance is not a result reflection. It is not only the relevance between the function of final system (the provided product and service amount) and results (the result that can effectively satisfy the expected missions and targets); but also contains the strategic implementation, management control, and comprehensive ability of project management during the implementation process of education e-government. Wherein, it includes the efficiency of operation and the ability of sustainable development.

In terms of the education e-government evaluation in college and university, the determination of e-government level can be transformed from "focus on input" to "focus on performance", which has important policy guidance and practical value. The evaluation on "performance" can help us lead the education information to an efficient, competitive, and sustainable development direction; it can help the education information cooperate with the overall strategy of colleges and universities; help school leaders to correctly recognize and implement the information in colleges and universities, thus achieving practical efficiency and avoiding waste.

\subsection{Education E-government Performance Evaluation in College and University}

E-government performance evaluation refers to the comprehensive assessment and evaluation for the construction, operation, management process, and results of college or university e-government by making use of certain verification methods, qualitative index and evaluation standards. In this way, it can promote the enhancement of its e-government management and application level.

\section{Design Principles of Index System}

\subsection{Scientific Principle}

The index system should reflect science, and it must abide by the laws and characteristics of education e-government. Meanwhile, it should accurately reflect the basic features of evaluation objects, as well as the actual level and effect during the process with the attitude of being practical and realistic. The science of 
index includes coincidence, independence, objectiveness, and coordination.

\subsection{Guiding Principle}

Evaluation index system plays a guiding role in enhancing education E-government performance. Therefore, when we are establishing index system, we should observe the selection of index factors, the connotation of factors, as well as the determination of evaluation standard. Meanwhile, we should emphasize the characteristics of education e-government and the main factors that influence the education e-government. It should reflect the basic requirements of education e-government, and also make all the factors the self-checking dimensions in the school, as well as the targets can be achieved in recent period. Meanwhile, through the evaluation factors of the evaluation index system and the weight distribution, it should guide all the teachers and students to reach the targets of education e-government.

\subsection{Feasible Principle}

The education E-government involves wide areas, while the index system cannot involve all aspects. Therefore, we need to analyze carefully, and master the main contradiction. We need to ensure the integrity of the index system, and simplify the index factors to the largest extent, to make it easier to be operated, but not being away from the actual situation of the school. Each evaluation index should be concrete and specific in content, independent in connotation and extension, distinct in wording, and clear in semantic meaning. It is the regulation for action and operation, and the data collection is simple, and with stable data source, as well as standard data and consistent range. Therefore, it is convenient for evaluators to observe and measure. To sum up, formulating index system can measure the e-government performance of education, and provide convenience to the implementation during evaluation process.

\subsection{Open and Adaptive Principle}

The education e-government is a dynamic development course, so the E-government performance evaluation of education should be continuously improved and consummated along the course of development. Therefore, we should appropriately revise the established evaluation index system after applying for certain period in accordance with the development and requirement of the situation, so as to enhance the consistency between the E-government evaluation of education and the E-government target of education, and also improve the efficiency of the E-government evaluation in education.

\subsection{Systematic Principle}

The evaluation index system should satisfy the completeness of index. That is to say, the formulated e-government performance evaluation index system of education should not neglect any important information, but comprehensively, systematically, and naturally reflect or cover all the factors of e-government performance in education. If the evaluation index system is more comprehensive and systematic, it can reflect the nature of evaluation objects better, and the evaluation result will be more accurate. However, it should be remembered that we cannot get away from reality for over pursuing completeness. When designing the evaluation index system, we should determine the factors that can best reflect the attributions off evaluation objects and best satisfy the requirements of evaluation targets as indexes in line with the characteristics of evaluation objects and the specific evaluation targets. On the other hand, some unimportant and non-essential evaluation factors can be neglected or abandoned. In this way, we can better combine completeness and focus.

\section{Construction Process of Evaluation Index System}

\subsection{Target Decomposition}

First, the evaluation targets can be decomposed. The general targets can be divided into several sub targets (primary index). According to the complexity of sub targets, some of the sub targets can be further decomposed to several sub sub targets (secondary index). Then, we should carefully analyze all the sub sub targets (sub targets) and all the involved factors (tertiary index), and then list them out without any 
omission.

\subsection{Classification and Induction}

Through induction and deduction, all the factors should be selected and refined. By getting rid of the nonessential and unimportant factors, while merging the same types of factors, and adjusting contradictory factors or the factors with causal-effect relationship, it will form the primary index framework.

\subsection{Comparison and Synthesis}

Through comprehensively applying literature research method, comparative research method, interview method, induction and deduction method, we should comparatively analyze and refer to the evaluation indexes generated through above analysis with the existing evaluation indexes, thus obtaining more comprehensive, objective, and accurate evaluation indexes. Therefore, the primary index framework will be shaped.

\subsection{Inspection and Revision}

Select appropriate evaluation target to conduct test in small scale. Meanwhile, revise the evaluation index in accordance with the test results.

\subsection{Verification and Perfection}

By making use of Delphi method, and through rounds of questionnaire surveys and expert consultation, the index framework will be determined. Then, the investigated factors can be further analyzed and selected. Through expert judgment, the investigated factors will be sorted, to determine the key investigated factors.

\subsection{Weight Determination}

By making use of subjective weighting method and objective weighting method, the index weight may be determined, and the complete index system may be obtained at last.

\section{Dimensional Analysis for Evaluation Index}

\section{System}

The following part will specifically analyze the four primary indexes to analyze e-government performance evaluation of education, which mainly collects data and judge the value from the following four evaluation dimensions.

(1) Information infrastructure

Information infrastructure includes the per capita possession amount of computer products, the proportion of computer network, the network performance level, information safety facilities, and other secondary indexes.

(2) Application system

The application system includes construction of office and business application system, construction of government information sources and basic data, construction of government information disclosure, construction of portal website, authorized software, and other secondary indexes.

(3) Cost and income

The cost and income includes the secondary indexes like hardware, software, personnel training, and operation input, enhancement of business quality and efficiency, reduction rate of administrative expense, improvement of scientific decisions, satisfactions from teachers, students, and the society.

(4) Safeguard system

The safeguard system includes the secondary indexes like views and talent environment, information planning and implementation level, standard application, as well as technical support and security safeguard.

\section{Methods to Obtain and Process Index Data}

\subsection{Methods to Obtain Index Data}

(1) Questionnaire

According to specific evaluation index, we should design effective questionnaire, to obtain evaluation index data.

(2) Expert evaluation

Based on experts' experience and knowledge, the qualitative index data can be obtained.

(3) Collective discussion and personal interviews

Collective discussion and personal interviews 
mainly invite related responsible persons from the evaluated objects, as well as the personnel from construction and operation management departments to make evaluation.

(4) Archives consultation

According to the data filled in the questionnaire, we should refer to the archives from the evaluated organizations.

(5) On-site verification

According to the data filled in the questionnaire, we should verify the authenticity of the data on the site.

(6) User evaluation

\subsection{Data Processing}

Data can be divided into quantitative data and qualitative data, so we should often scientifically process data when applying the application model.

(1) Qualitative index processing

Qualitative data processing usually adopts questionnaire method and field inspection. In order to avoid errors caused by subjective judgment, and increase the accuracy of qualitative indicators, we can use membership value assignment method, and classify qualitative indexes into several classes, which are 1-10 respectively.

(2) Quantitative index processing

Because contents and dimensions of each qualitative index are different, these indexes should undergo dimensionless processing, and transfer the original quantitative index value to evaluation value.

\section{Index Weight Determination through AHP Method}

Index weight is an important component of index system, which decides the importance degree of each index in the whole evaluation system, and also determines that scientific and reasonable weight is a vital item in comprehensive evaluation. This paper adopts the analytic hierarchy process (AHP) on the basis of Delphi Method to determine the weight. AHP was put forward by the famous American operational scientist T. L. Satty in the 1970s, and it was a systematic and hierarchical analysis method by combining qualitative and quantitative factors. The basic thinking of AHP is to decompose the factors relating to decision into targets, standards, and programs. Based on this, we can conduct qualitative and quantitative analysis.

\subsection{Establish Hierarchy Frame Model}

The key of AHP lies in establishing hierarchical model. In order to solve modeling problems, T.L.Satty divided the factors included in the problems into several layers, which may be generally classified as the highest layer, the middle layer, and the lowest layer. According to the principle of AHP and the above analysis for evaluation index system, we can obtain the hierarchical model of evaluation shown.

\subsection{Construct Judgment Matrix}

After establishing hierarchical structural model, it should point out the relative importance of all the factors in each layer regarding to the related factors on the previous layer, and then mark the value. Then, it can construct the judgment matrix, which is a key step of AHP. In order to get the value of each element in the matrix, the experts should first compare all the factors and then give out the judgment value. Then, according to the nine-level scales, the judgment result will be quantified, thus forming the comparative judgment matrix. Through applying Delphi method, we should organize experts to conduct questionnaire method. And through collecting the contents of the investigation tables from experts, we have obtained five judgment matrixes (for limited length of the paper, they are not listed out).

\subsection{Single Hierarchical Arrangement}

Single hierarchical arrangement is the weight from all the indexes of a certain layer to the relative importance of indexes at the previous layer. The commonest calculating methods include square root method, sum, characteristic root method, and least square method. However, the least square method is adopted in this paper, and the specific calculation steps are shown as follows:

Providing: 


$$
z=\sum_{i=1}^{n} \sum_{j=1}^{n}\left[\ln a_{i j}-\ln \left(\frac{\bar{w}_{i}}{\bar{w}_{j}}\right)\right]^{2}
$$

According to the fair and just principles, several judges (or several group judges) are selected to take part in the evaluation, so the factors of the judgment matrix $a_{i j}$ have several values. Therefore, the formula (1) can be expanded as:

$$
z=\sum_{i=1}^{n} \sum_{j=1}^{n} \sum_{k=1}^{b}\left(\ln a_{i j k}-\ln \bar{w}_{i}+\ln \bar{w}_{j}\right)^{2}
$$

Wherein, $b$ is the constant, namely the number (or group number) of the judges.

Obtain the partial derivative of

$\bar{w}_{i}(i=1,2, \cdots, n)$ for both sides of the formula (2),

which can be simplified as:

$$
n \ln \bar{w}_{i}-\sum_{i=1}^{n} \ln \omega_{i}=\frac{1}{b} \sum_{j=1}^{n} \sum_{k=1}^{b} \ln a_{i j k}
$$

From formula (3), we can get:

$$
\bar{w}_{i}=\left(\prod_{k=1}^{b} \prod_{j=1}^{n} \omega_{j}\right)^{\frac{1}{b n}}\left(\prod_{k=1}^{b} \prod_{j=1}^{n} a_{i j k}\right)^{\frac{1}{b n}}
$$

At last, normalize the vector $\bar{W}=\left(\overline{W_{1}}, \overline{W_{2}}, \cdots, \overline{W_{n}}\right)$.

$$
w_{i}=\frac{\overline{w_{i}}}{\sum_{i=1}^{n} w_{i}}=\frac{\left(\prod_{k=1}^{b} \prod_{j=1}^{n} a_{i j k}\right)^{\frac{1}{b n}}}{\sum_{i=1}^{n}\left(\prod_{k=1}^{b} \prod_{j=1}^{n} a_{i j k}\right)^{\frac{1}{b n}}}
$$

Then, $W=\left(W_{1}, W_{2}, \cdots, W_{n}\right)^{T}$ is the characteristic vector.

Therefore, we can obtain the following weight of primary index and secondary index, which is shown as follows.

$$
\begin{aligned}
& \omega_{1}=0.356, \omega_{2}=0.236, \omega_{3}=0.215, \omega_{4}=0.193 \\
& \omega_{11}=0.4235, \omega_{12}=0.2271, \omega_{13}=0.2271, \omega_{14}=0.1223 \\
& \omega_{21}=0.491, \omega_{22}=0.232, \omega_{23}=0.093, \omega_{24}=0.138, \omega_{25}=0.046 \\
& \omega_{31}=0.263, \omega_{32}=0.475, \omega_{33}=0.058, \omega_{34}=0.093, \omega_{35}=0.111 \\
& \omega_{41}=0.055, \omega_{42}=0.565, \omega_{43}=0.117, \omega_{44}=0.263
\end{aligned}
$$

In this way, we can obtain a complete evaluation index system, which is shown as Table 1 .

\section{Future Research Direction}

Education e-government performance evaluation is a very complicated issue, especially for the determination of the evaluation index and the determination of each index weight. The education e-government performance evaluation index system of college and university with different types and in different areas is different. However, AHP can make our thinking process mathematical, modeling, systematic, and standardized, so making decisions through AHP can greatly enhance the efficiency of the decision making. Nevertheless, during the process of applying AHP, no matter establishing hierarchical structure or constructing judgment matrix, people's subjective judgment, selection, and preference has great influence on the result. Therefore, it is very subjective to make decisions by making use of AHP. In other words, how to overcome the subjective influence, how to make use of group judgment, and how to combine AHP with other decision making methods are the future research directions. 
Table 1 Education E-government Performance Evaluation Index System in College and University

\begin{tabular}{|c|c|c|c|}
\hline \multicolumn{2}{|c|}{ Primary Index } & \multicolumn{2}{|l|}{ Secondary Index } \\
\hline Index Item & Weight & Index Item & Weight \\
\hline \multirow{4}{*}{$\begin{array}{l}\text { Information } \\
\text { Infrastructure }\end{array}$} & \multirow{4}{*}{0.356} & per capita possession amount of computer product & 0.4235 \\
\hline & & computer network proportion & 0.2271 \\
\hline & & network performance level & 0.2271 \\
\hline & & information safety insurance & 0.1223 \\
\hline \multirow{5}{*}{ Application System } & \multirow{5}{*}{0.236} & construction of office and business application system & 0.491 \\
\hline & & construction of government information resource and basic data & 0.232 \\
\hline & & construction of government information public & 0.093 \\
\hline & & portal website construction & 0.138 \\
\hline & & authorized software & 0.046 \\
\hline \multirow{5}{*}{ Cost and Income } & \multirow{5}{*}{0.215} & software, hardware, personnel training, and operation input & 0.263 \\
\hline & & office quality and efficiency enhancement & 0.475 \\
\hline & & reduction ratio of administrative expense & 0.058 \\
\hline & & enhancement of scientific decision level & 0.093 \\
\hline & & satisfaction degree of teachers, students, and the society & 0.111 \\
\hline \multirow{4}{*}{ Safety System } & \multirow{4}{*}{0.193} & views and talent environment & 0.055 \\
\hline & & information planning and implementation level & 0.565 \\
\hline & & standard application & 0.117 \\
\hline & & technical support and security safeguard & 0.263 \\
\hline
\end{tabular}

\section{Acknowledgment}

This study is supported by the construct program of the key discipline in human province, Hunan Philosophy and social science program(NO: 13YBB205), Hunan science and technology program(NO:2014SK3229), Hunan province ordinary university teaching reform project(xiangjiangtong[2013]NO:223),China.

\section{References}

1. OECD.www.oecd.org.(2001)

2. UN.www.un.org.8(2003)

3. Tang Jun. J.E-Government,24(2005)

4. LeiZhanbo,JiangXiaofang. J. Intelligence, 12(2006)

5. Zhang Chengfu, Tang Jun. J. E-government, 2005,(24).

6. Yang Yunfei, Bai Qinghua. J. Comp. Appl. Soft, 2004,(8).

Evaluation(Publishing House of Electronics Industry,2005)
7. Peng Xizheng. J. Info. Cons., 10(2005)

8. Ma Lianjie, Hu Xinli, Zhang Xiaolian, et. al. J. Hubei Social Science,11(2005)

9. Liu Tenghong, Liu Ronghui, Zhao Peng. J.Journal of Wuhan University of Technology (Information and Management Engineering),3(2004)

10. Wang Xiezhou, Chen Yanhong. J. Archives Science Study,6(2004)

11. Zhijuan Liu,Jiaming Zhong.J.IERI Procedia,2(2012)

12. Du Dong, Pang Qinghua. Modern Comprehensive Evaluation Methods and Case Selection (Tsinghua University Press,2006)

13. ZhangXiaoming. J.Terminology Standardization \& Information Technology, 2(2005)

14. An Hong,Gao Xuedong,Zeng Dehua,etc.J.Transactions of Beijing Institute of Technology,27,8(2007)

15. Zhang Haofeng,Kong Fanshi. Educational Information 